\title{
Propiedades psicométricas del Inventario de Afrontamiento COPE Breve en una muestra de cuidadores primarios informales de pacientes con cáncer en población mexicana ${ }^{1}$
}

\author{
Psychometric properties of the Brief COPE Inventory \\ in a sample of informal primary caregivers of cancer patients \\ in the Mexican population
}

\author{
Javier Núñez Hernández 2 , Oscar Galindo Vázquez 3 , Frank J. Penedo ${ }^{4}$, \\ German Calderillo Ruiz ${ }^{5}$, Abelardo Meneses García ${ }^{6}$, \\ Ángel Herrera Gómez ${ }^{7}$ y Abel Lerma ${ }^{8}$
}

Citación: Núñez H., J., Galindo V., O., Penedo, F.J., Calderillo R., G., Meneses G., A., Herrera G., Á. y Lerma, A. (2021). Propiedades psicométricas del Inventario de afrontamiento COPE Breve en una muestra de cuidadores primarios informales de pacientes con cáncer en población mexicana. Psicología y Salud, 31(1), 143-153. https://doi.org/10.25009/pys.v31i1.2684.

RESUMEN

\begin{abstract}
Introducción: Los cuidadores primarios informales de pacientes con cáncer constituyen una importante población en el sistema de salud, por lo que es importante conocer cómo afrontan esa circunstancia. Objetivo: Determinar las propiedades psicométricas del Inventario de Afrontamiento COPE Breve en una muestra de cuidadores primarios informales de pacientes con cáncer en población mexicana. Metodología: La muestra estuvo conformada por 180 participantes de entre 18 a 67 años, de un hospital público de tercer nivel de la Ciudad de México. Análisis estadísticos: Se realizó un análisis mediante el método de componentes principales y rotación varimax, y posteriormente un análisis factorial confirmatorio. Resultados: Se obtuvieron propiedades psicométricas adecuadas, con una estructura factorial similar a la de la versión original. La consistencia interna fue de 0.80 , con una varianza explicada de $55.74 \%$. El análisis factorial confirmatorio mostró indicadores y ajuste adecuados de la estructura del modelo. Discusión: El Inventario de Afrontamiento COPE Breve es un instrumento válido y confiable. Se recomienda su uso en atención clínica e investigación en una población que puede requerir atención psicológica durante el proceso de cuidado del paciente con cáncer.
\end{abstract}

Palabras clave: Afrontamiento; Análisis factorial; Cuidador primario informal; Cáncer; Población mexicana.

\footnotetext{
${ }^{1}$ Esta investigación estuvo financiada por el proyecto CONACYT "Efectos de una intervención cognitivo-conductual sobre la adhesión al tratamiento, tabaquismo y calidad de vida, mediante la modificación de los estilos de afrontamiento en pacientes con cáncer de mama en inicio de tratamiento médico", PN 2015/1715.

${ }^{2}$ Universidad Latina, Campus Sur, Av. Pedro Henríquez Ureña 173, Los Reyes, Coyoacán, 04330 Ciudad de México, México.

${ }^{3}$ Servicio de Psicooncología del Instituto Nacional de Cancerología, Calle Av. San Fernando No. 22, Col. Sección XVI, 14080 Ciudad de México, México, correo electrónico: psigalindo@yahoo.com.mx.

${ }^{4}$ College of Arts and Sciences, Miller School of Medicine, Miami University, $1600 \mathrm{NW} 10^{\text {th }}$ Ave \#1140, 33136 Miami, FL, Estados Unidos.

${ }^{5}$ Subdirección de Medicina Interna del Instituto Nacional de Cancerología.

${ }^{6}$ Dirección General del Instituto Nacional de Cancerología.

${ }^{7}$ Dirección General Adjunta Médica del Instituto Nacional de Cancerología.

${ }^{8}$ Instituto de Ciencias de la Salud (ICSa), Universidad Autónoma del Estado de Hidalgo, Carretera Pachuca-Actopan, camino a Tilcuautla s/n, 42160 Pueblo San Juan Tilcuautla, Hgo., México.
} 


\begin{abstract}
Background: Informal primary caregivers of cancer patients are an important population in the health system, since they carry out tasks of family health care, so it's important to know how they cope with these circumstances. Objective: Determine the brief COPE coping inventory psycometric properties for a sample of informal primary caregivers of cancer patients in the Mexican population. Methodology: The sample was 180 participants between 18 to 67 years old in a third level public hospital in Mexico City. Statistical analysis: An analysis was performed using the principal components and varimax rotation method. Subsequently, a confirmatory factor analysis was performed. Results: Adequate psychometric properties were obtained with a factorial structure similar to the original version. The global internal consistency was 0.80 and an explained variance of $55.74 \%$ of variance. Confirmatory factor analysis shows adequate indicators and the adjustment of the model structure. Discussion: The brief COPE coping inventory is a valid and reliable instrument, and the use in clinical care and research is recommended in a population that may require psychological attention during the process of caring for the cancer patient.
\end{abstract}

Key words: Coping: Primary caregiver: Cancer: Factor analysis: Mexican population.

\section{INTRODUCCIÓN}

$\mathrm{S}$ egún datos del National Alliance for Caregiving (2015), los cuidadores primarios informales (CPI en lo sucesivo) brindaron la casi totalidad de los cuidados de largo plazo a sus familiares. De acuerdo con este estudio, se estimaba que a fines del año 2014 un total de 43.5 millones de personas afirmaron brindar ayuda a una persona enferma, ya fuesen niños o adultos; de estos, 34.2 millones atendieron a pacientes de 50 años o más, y uno de cada diez a un adulto mayor de 75 años. En promedio, habían desempeñado su función durante cuatro años, y una cuarta parte de ellos había brindado la atención durante cinco años o más (National Alliance for Caregiving, 2015).

Debido a las reformas realizadas entre los años 1980 y 1990 en el sistema de salud, se generó una reducción en estos servicios (Papastavrou,
Charalambous y Tsangari, 2009; Papastavrou et al., 2011), por cual, hubo un cambio en el esquema, pasándose del cuidado hospitalario al ambulatorio (Ramírez, Addington-Hall y Richards, 1998), lo que trajo consigo una mayor presión y más responsabilidades en el cuidado para los familiares del paciente, normalmente los cónyuges, hijos, hermanos o padres (Larrañaga et al., 2008; Nijboer et al., 1998; Papastavrou et al., 2011; Printz, 2011; Rhee et al., 2008). De acuerdo a Ullgren, Tsitsi, Papastavrou y Charalambous (2018), el cuidado de un familiar puede extenderse a lo largo de varios años, incluso durante el periodo de sobrevivencia.

Un sector de la población muy afectado por el estrés derivado de las actividades de cuidado son los CPI. De acuerdo con Feldberg et al. (2011), los CPI son los principales responsable del cuidado no profesional del paciente; residen en su gran mayoría en el mismo domicilio del enfermo, deben estar disponibles para satisfacer sus demandas y no reciben retribución económica alguna por las funciones que desempeñan.

Diversos estudios indican que las mujeres son la principal fuente de cuidados de los familiares, siendo por lo general la esposa o las hijas, usualmente sin empleo, de bajo nivel socioeconómico y educativo y que dedican de seis a doce horas diarias a cuidar a su familiar, considerando que conforme progresa la enfermedad viven permanentemente en el mismo domicilio que el receptor del cuidado (García, Mateo y Maroto, 2004; Martínez, Díaz y Gómez, 2010; National Alliance for Caregiving, 2015; Nigenda, López, Matarazzo y Juárez, 2002; Vaquiro y Stiepovich, 2010; Wenk y Monti, 2006). Ello implica que los CPI encaren responsabilidades para las que no están calificados médica, práctica ni emocionalmente (Alexander, 2010; Hudson y Payne, 2009; Kristjanson y Aoun, 2004; Ullgren et al., 2018). Estas tareas pueden cambiar o hacerse más complejas conforme avanza la enfermedad del paciente (Papastavrou et al., 2009; Ullgren et al., 2018), y algunas otras son tareas que el equipo de salud realizaba anteriormente (Papastavrou et al., 2009; van Ryn et al., 2011), como por ejemplo administrar medicamentos, programar y acompañar al paciente a sus visitas médicas, tomar decisiones sobre el 
tratamiento o el manejo del mismo y darle apoyo emocional (Adelman, Tmanova, Delgado, Dion y Lachs, 2014; Lamura et al., 2006).

Por otro lado, los CPI ayudan con las tareas domésticas, apoyan en la alimentación del paciente, en la gestión de recursos económicos y en el transporte (Osse, Vernooij-Dassen, Schadé y Grol, 2006). Frecuentemente tales actividades originan cambios en diversos aspectos de su propia vida, como en sus rutinas y hábitos, y los llevan a afrontar en ocasiones pérdidas laborales, a privarse de sus amistades e incluso a descuidar su salud en el proceso de cuidar a su familiar, a lo que se agrega un proceso de sobrecarga que puede afectar su calidad de vida y aumentar sus niveles de ansiedad y depresión (Alfaro et al., 2008; Vanderwerker, Laff, Kadan-Lottick, McColl y Prigerson, 2005).

La forma en la que los CPI afrontan la situación de cuidar a su paciente es esencial, ya que los estilos de afrontamiento determinan la forma en la que el cuidador aborde los problemas que se le presentan (McMillan et al., 2006; Carver, Scheie, y Weintraub, 1989; Lazarus y Folkman, 1984), considerando que el afrontamiento es entendido como los esfuerzos cognitivos y conductuales constantemente cambiantes que se utilizan para manejar las demandas externas o internas que exceden o desbordan los recursos del individuo (Lazarus y Folkman, 1984).

Hay pocos instrumentos validados que midan el afrontamiento en CPI de pacientes adultos con cancer (Vázquez et al., 2017). En aquellos validados para población mexicana únicamente se identificó el diseñado por Espinoza, Reyes, Velasco, Bravo y Ramírez (2017) utilizando el modelo de afrontamiento de Lazarus y Folkman (1984), el cual, sin embargo, fue elaborado específicamente para CPI de pacientes menores de edad con cancer.

En este contexto, la escala de afrontamiento COPE (Carver, 1997) es un instrumento sumamente utilizado en CPI en sus versión original de quince subescalas y sesenta reactivos (Carver et al., 1989), desarrollada a partir de la teoría sobre el estrés de Lazarus y Folkman (1984) y del modelo de autorregulación conductual de Carver y Scheier (1981, 1990).

Por su parte, el COPE Breve, elaborada por Carver (1997) se ha traducido a diferentes idiomas y se han analizado sus propiedades psicométricas en población general (Doron et al., 2014; Kapsou, Panayiotou, Kokkinos y Demetriou, 2010; Maroco, Campos, Bonafé, Vinagre y Pais-Ribeiro, 2014; Ruiz et al., 2015), estudiantes (Lee y Liu, 2001; Morán, Landero y González, 2010), cuidadores de pacientes con VIH-sida (Kimemia, Asner-Self y Daire, 2011), pacientes con VIH-sida (Mohanraj et al., 2015), mujeres con cáncer de mama (Ben-Zur, Gilbar y Lev, 2001; Vargas, Herrera, Rodríguez y Sepúlveda, 2010; Yusoff, Low y Yip, 2010), pacientes con cáncer y sus cuidadores primarios (Baumstarck et al., 2017), padres de niños con enfermedad crónica (Hastings et al., 2005; Zelikovsky, Schast y Jean-Francois, 2007) y adultos con trastornos de ansiedad (Pozzi et al., 2015). De esos estudios se han llevado a cabo diversos análisis factoriales confirmatorios con estructuras similares a la de la versión original (Baumstarck et al., 2017; Doron et al., 2014; Mohanraj et al., 2015).

En este contexto resulta indispensable disponer de instrumentos válidos y confiables de evaluación psicológica que haga posible conocer la manera en que afrontan los CPI el cuidado de su familiar, por lo que el objetivo del presente estudio fue obtener las propiedades psicométricas del Inventario de Afrontamiento COPE Breve para CPI de pacientes con cáncer adultos en población mexicana.

\section{MÉTODO}

\section{Participantes}

Se incluyeron 180 participantes seleccionados por muestreo no probabilístico, cuyas características se muestran en la Tabla 1.

Los CPI fueron seleccionados a partir de los siguientes criterios: ser acompañante que de forma verbal indicara ser el principal proveedor de cuidados al paciente, saber leer y escribir y no recibiera remuneración económica por el cuidado provisto; se excluyeron los cuidadores con problemas auditivos o visuales severos, o que tuviesen un adiestramiento profesional o técnico en cuidados de pacientes, y se eliminaron los CPI que desistieran de participar durante la aplicación de los instrumentos o después de la misma. 
Tabla 1. Descripción de la muestra.

\begin{tabular}{|c|c|c|}
\hline CARACTERÍSTICAS & f & $\%$ \\
\hline $\mathbf{N}$ & 180 & 100 \\
\hline \multicolumn{3}{|c|}{ Edad (años cumplidos): 18 a $67(\mathrm{x}=41.5)$} \\
\hline \multicolumn{3}{|c|}{ Sexo } \\
\hline Hombres & 50 & 27.8 \\
\hline Mujeres & 130 & 72.2 \\
\hline \multicolumn{3}{|l|}{ Paternidad } \\
\hline Sí & 131 & 72.8 \\
\hline No & 49 & 27.2 \\
\hline \multicolumn{3}{|l|}{ Lugar de residencia } \\
\hline Ciudad de México & 90 & 50 \\
\hline Interior de la República & 90 & 50 \\
\hline \multicolumn{3}{|l|}{ Estado civil } \\
\hline Casado(a) o con pareja & 113 & 62.7 \\
\hline Sin pareja & 67 & 37.3 \\
\hline \multicolumn{3}{|l|}{ Situación laboral } \\
\hline Hogar & 61 & 33.9 \\
\hline Empleado(a) & 36 & 20 \\
\hline Comerciante & 24 & 13.3 \\
\hline Autoempleo/Oficio & 19 & 10.6 \\
\hline Jubilado(a) & 7 & 3.9 \\
\hline Desempleado(a) & 5 & 2.8 \\
\hline \multicolumn{3}{|l|}{ Nivel de estudios } \\
\hline Primaria & 15 & 8.3 \\
\hline Secundaria & 25 & 25.0 \\
\hline Bachillerato & 55 & 30.6 \\
\hline Licenciatura & 57 & 31.7 \\
\hline Posgrado & 8 & 4.4 \\
\hline \multicolumn{3}{|c|}{ Enfermedad diagnosticada } \\
\hline Sí & 31 & 17.2 \\
\hline No & 149 & 82.8 \\
\hline \multicolumn{3}{|l|}{ Meses de cuidado } \\
\hline 1 a 15 & 102 & 56.7 \\
\hline 16 a 43 & 45 & 25 \\
\hline 44 o más & 33 & 18.3 \\
\hline \multicolumn{3}{|c|}{ Reciben ayuda para cuidar al paciente } \\
\hline Sí & 117 & 65 \\
\hline No & 63 & 35 \\
\hline \multicolumn{3}{|c|}{$\mathrm{N}^{\circ}$ de personas que ayudan al CPI } \\
\hline Una & 53 & 29.4 \\
\hline Dos & 35 & 19.4 \\
\hline Tres & 18 & 10 \\
\hline Cuatro o más & 11 & 6.2 \\
\hline
\end{tabular}

\section{Procedimiento}

\section{Etapa 1. Adaptación cultural.}

El instrumento fue traducido al español por un psicólogo profesional en el área de la salud, versión que se sometió a un jueceo por expertos para realizar las modificaciones pertinentes en cuanto a claridad, comprensión y pertinencia; finalmente, se efectuó un piloteo con 30 CPI que se encontraban en las salas de espera de las unidades funcionales, mediante el cual se evaluó la dificultad, palabras confusas, difíciles de comprender y lenguaje ofensivo en las instrucciones, los reactivos y las opciones de respuesta (Hernández, Fernández y Baptista, 2014).

\section{Etapa 2. Aplicación del instrumento.}

Una vez que se sabía que los CPI cumplían con los criterios de inclusión, se les abordó en las distintas salas de espera de las unidades funcionales de la institución para invitarlos a participar en el estudio, cuyo objetivo era conocer la forma en que habían afrontado el hecho de cuidar a su paciente. Se les solicitó firmar la hoja de consentimiento informado aseverándoles que la información que proporcionaran sería totalmente confidencial. Una vez que comprendían y firmaban dicho documento, procedían a responder el COPE.

Dichos CPI fueron evaluados en consulta externa antes o después de la atención de su familiar en las diferentes unidades funcionales del INCan. El proyecto fue aprobado por el Comité de Investigación y Ética del Instituto Nacional de Cancerología (INCan).

\section{Instrumentos}

\section{Cedula de identificación.}

Se incluyó una cédula en la cual se recabaron algunas variables sociodemográficas (edad, sexo, estado civil, escolaridad, paternidad y nivel estudios) y clínicas (enfermedad diagnosticada en el paciente, meses de cuidado y ayuda recibida para cuidar a su familiar). 
Inventario de Afrontamiento COPE Breve.

El COPE Breve está constituido por 28 reactivos agrupados en 14 subescalas: afrontamiento activo, planificación, apoyo instrumental, uso de apoyo emocional, autodistracción, ventilación, desconexión conductual, reformulación positiva, negación, aceptación, religión, uso de sustancias, humor y autoculpa. Dichos ítems están planteados en términos de las acciones o pensamientos utilizados como formas de afrontamiento, y cada uno contiene cuatro opciones de respuesta ("Nunca", "Poco", "Casi siempre" y "Siempre"), referidas a la frecuencia con que la persona lleva a cabo una acción o tiene un pensamiento. Los puntajes altos indican un uso más frecuente de ese estilo de afrontamiento. Los coeficientes de confiabilidad de las 14 subescalas originales tienen valores alfa de Cronbach de entre .50 y .90 (Carver, 1997), lo que indica una consistencia interna adecuada. El instrumento COPE Breve es uno de los instrumentos de afrontamiento más usados a nivel mundial de acuerdo a algunas revisiones sistemáticas (Krägeloh, Chai, Shepherd y Billington, 2012; Kvillemo y Bränström, 2014).

\section{Análisis estadísticos}

\section{Análisis factorial exploratorio (AFE).}

El análisis de consistencia interna del instrumento se llevó a cabo a través del programa estadístico SPSS para Windows, v. 21; se realizó asimismo un análisis factorial exploratorio (AFE) con rotación varimax, y se determinó su consistencia interna mediante el coeficiente alfa de Cronbach, adoptándose un nivel de significancia estadística de $p<.05$ (Gardner y Escalona, 2003).

\section{Análisis factorial confirmatorio (AFC).}

A partir del análisis exploratorio del instrumento, se evaluó el ajuste de seis factores mediante un análisis factorial confirmatorio (AFC), usando para ello el método de máxima verosimilitud, mismo que incluyó los pasos de identificación y especificación del modelo, la estimación de parámetros estandarizados (correlaciones $\mathrm{R}^{2}$, covarianzas, ín- dices de modificación y proporciones críticas de las diferencias), y por último, la evaluación del ajuste mediante la observación de límites aceptables de los estimadores, así como la no colinealidad en las variables medidas. Se estimaron los siguientes índices de ajuste global $\mathrm{X}^{2}$ y la razón $\mathrm{X}^{2} / \mathrm{gl}$, los índices de bondad de ajuste (GFI) y sus complementos (AGFI, TLI), al igual que el índice de bondad de ajuste comparativo (CFI), que es el mejor indicador para muestras iguales o superiores a 200, y por último la raíz cuadrada media del error de aproximación (RMSEA) (Byrne, 2010; George y Mailery, 2001; Hu y Bentler, 1998; Kline, 2005; Ullman, 2006).

\section{RESULTADOS}

\section{Análisis factorial exploratorio (AFE).}

Después de realizar la prueba $t$ para muestras independientes, según el proceso del análisis factorial se determinó que cuatro de los ítems de la escala (4 y 11: uso desustancias; 13: autoculpa, y 16: desconexión conductual) no contaban con las características apropiadas para ser considerados en el análisis de fiabilidad. Por consiguiente, se corrió el análisis con 24 de los reactivos y considerando .50 como el punto de corte (Hair, Anderson, Tatham y Black, 1999), quedando de esta forma 22 reactivos (se eliminaron el ítem 7 , "afrontamiento activo", y 20, "aceptación”) que, de acuerdo al AFE, mostraron seis factores, con un coeficiente alfa de .80 y una varianza explicada de $55.74 \%$. El resultado del estadístico KMO fue de .73 y la prueba de esfericidad de Bartlett resultó significativa $\left(\mathrm{X}^{2}=1313.83 ; g l=276 ; p=.001\right)$. Asimismo, las cargas factoriales para cada factor fueron aceptables (Tabla 2).

\section{Análisis factorial confirmatorio (AFC).}

La razón chi cuadrado/gl de ajuste global (97.9, $p=0.002$ ) indica errores casi nulos en las varianzas y covarianzas del modelo ajustado a esta población. El principal índice de bondad de ajuste $(\mathrm{GFI}=0.930)$ y el comparativo $(\mathrm{CFI}=0.951)$ 
Tabla 2. Estructura factorial del COPE Breve.

\begin{tabular}{|c|c|}
\hline $\begin{array}{l}\text { Alfa de Cronbach global } \alpha=.80 \\
\text { Varianza explicada }=55.74 \%\end{array}$ & $\begin{array}{c}\text { Carga } \\
\text { factorial }\end{array}$ \\
\hline Factor 1. Apoyo emocional / Apoyo instrumental & .850 \\
\hline 10. Recibo ayuda y consejos de otras personas (como familiares y amigos). & .867 \\
\hline 5. Recibo apoyo emocional de otras personas (como familiares y amigos). & .809 \\
\hline $\begin{array}{l}\text { 15. Recibo consuelo y comprensión de alguien más (como familiares } \\
\text { y amigos, por ejemplo). }\end{array}$ & .809 \\
\hline $\begin{array}{l}\text { 23. Trato de seguir consejos u obtener ayuda de otras personas } \\
\text { sobre lo que debo hacer para cuidar a mi paciente. }\end{array}$ & .589 \\
\hline Factor 2. Religión, aceptación y planificación & .700 \\
\hline 27. Rezo o medito para afrontar esta situación. & .793 \\
\hline 22. Trato de encontrar consuelo en mi religión o en mis creencias espirituales. & .757 \\
\hline 25. Pienso mucho sobre qué hacer para cuidar a mi paciente. & .592 \\
\hline 24. Aprendo a vivir con el hecho de que debo cuidar a mi paciente. & .530 \\
\hline Factor 3. Autodistracción y expresión emocional & .550 \\
\hline $\begin{array}{l}\text { 19. He estado haciendo algunas cosas para pensar menos en mi situación } \\
\text { (por ejemplo, ir al cine, ver televisión, leer, soñar despierto, dormir } \\
\text { o ir de compras). }\end{array}$ & .706 \\
\hline $\begin{array}{l}\text { 1. Trabajo o hago otras actividades para distraerme del cuidado } \\
\text { de mi paciente. }\end{array}$ & .587 \\
\hline $\begin{array}{l}\text { 21. Expreso mis sentimientos negativos (como tristeza, enojo o ansiedad) } \\
\text { respecto a la situación que vivo con mi paciente. }\end{array}$ & .519 \\
\hline $\begin{array}{l}\text { 9. Digo cosas para dejar que salgan mis sentimientos desagradables } \\
\text { (como enojo, frustración o estrés). }\end{array}$ & .504 \\
\hline Factor 4. Afrontamiento activo y desconexión conductual & .410 \\
\hline 14. Trato de pensar en un plan para cuidar a mi paciente. & .608 \\
\hline 2. Me esfuerzo por cuidar mejor a mi paciente. & .571 \\
\hline 6. Dejé de hacer frente al estrés que me provoca cuidar a mi paciente. & .545 \\
\hline Factor 5. Reformulación positiva y humor & .600 \\
\hline 18. Hago bromas para hacer más amena mi situación actual. & .741 \\
\hline 28. Me río de mi situación actual. & .696 \\
\hline $\begin{array}{l}\text { 12. Trato de ver el hecho de cuidar a mi paciente con una perspectiva } \\
\text { más positiva }\end{array}$ & .516 \\
\hline 17. Pienso que cuidar a mi paciente es una experiencia positiva. & .501 \\
\hline Factor 6. Negación y autoculpa & .600 \\
\hline 8. Me niego a aceptar que esto está pasando. & .746 \\
\hline 3. Me digo a mí mismo que esto no es real. & .742 \\
\hline 26. Me culpo por la enfermedad de mi paciente. & .592 \\
\hline
\end{tabular}

confirman el modelo como complejo y aceptable, mientras que los índices RMR $=0.051$ (próximo a 0 ) y RMSEA $=0.058$ lo ratifican ya que penalizan la complejidad, pero los índices se mantienen por debajo o muy próximos a 0.05 y 0.08 , respecti- vamente, por lo que el modelo es recursivo y se identifica correctamente. Las cargas factoriales y la varianza del error explicado por cada reactivo pueden observarse en la Figura 1. 
Figura 1. Modelo AFC del COPE Breve.

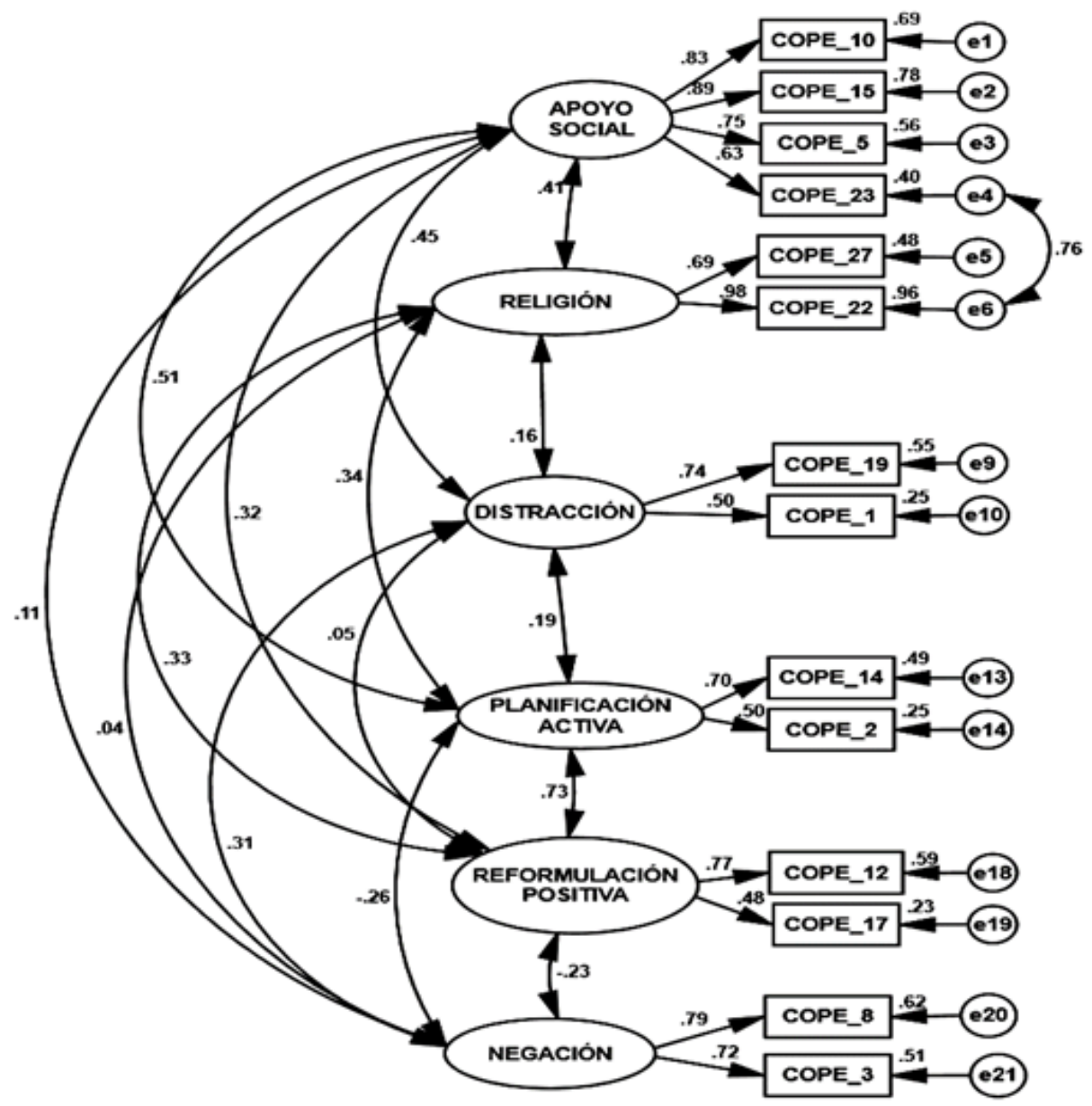

Chi cuadrada $=97.9,61 \mathrm{gl}, \mathrm{p}=0.002, \mathrm{CMIN} / \mathrm{gl}=1.606$; $\mathrm{RMR}=0.051, \mathrm{GFI}=0.930, \mathrm{AGFI}=0.879, \mathrm{TLI}=0.927, \mathrm{CFI}=0.951, \mathrm{RMSEA}=0.058(0.036-0.079)$. Hoelter (adecuación muestral), $p=0.01, \mathrm{n}=164$.

\section{DISCUSIÓN}

Los CPI desempeñan un papel significativo como principales figuras de apoyo, y el personal médico y paramédico es cada vez más consciente de esta realidad. Por tanto, es necesario involucrar a aquellos activamente en la prevención de respuestas negativas en cualquier momento, razón por la cual el control de sus emociones y comportamiento repercute sobre los pacientes de modo positivo (Ortega, Galindo y Meneses, 2018).

En este sentido, la muestra del presente estudio se compuso principalmente de CPI casados o con pareja, con paternidad satisfecha, la mayor parte en el primer año del cuidado de su paciente y reportando una ligera mayoría recibir ayuda para las labores de cuidado. Es de destacar que 17.2\% dijo sufrir alguna enfermedad, la que puede ser el producto de un subdiagnóstico ya que algunos CPI pueden presentar algún tipo de enfermedad sin estar diagnosticados médicamente.

Este es el primer informe que reporta la validez y confiabilidad del Inventario de Afrontamiento COPE Breve para CPI de pacientes con cáncer en población mexicana. Con ello se busca aportar evidencias a la literatura en el área mediante los presentes resultados. Debe decirse que la forma en que se distribuyeron los ítems fue similar a la estructura original propuesta por el autor (Carver, 1997).

Una contribución del presente estudio es el análisis confirmatorio, el cual no ha sido reportado de forma frecuente, en tanto que la versión mexicana del inventario COPE Breve ha demostrado una estructura teórica con seis factores de primer orden (apoyo social, religion, distracción, 
planificación activa, reformulacíon positiva y negación) y un factor general de segundo orden, con índices de bondad de ajuste adecuados y que se amolda apropiadamente a los datos empíricos de la muestra estudiada, que explican un suficiente porcentaje de la varianza y una adecuada confiabilidad, lo que ofrece evidencias de la calidad psicométrica de este instrumento.

Se identificaron seis factores, tal como ocurrió en una validación anterior (Su et al., 2015), quedando de la siguiente forma: primer factor: apoyo emocional y apoyo instrumental, denominado Apoyo social (Heydecke, Tedesco, Kowalski e Inglehart, 2004; Krägeloh et al., 2012; Pozzi et al., 2015; Radat et al., 2009), el cual ha demostrado ser un elemento predictor de adaptación psicológica ante eventos estresantes y adversos. E1 segundo factor se conformó exclusivamente por la subescala de Religión (Fillion, Kovacs, Gagnon y Endler, 2002; Kapsou, Panayiotou, Kokkinos y Demetriou, 2010; Ornelas et al., 2013; Pozzi et al., 2015), que se ha identificado como una forma de apoyo de que disponen las personas para superar situaciones estresantes o vistas como un desafío (Krägeloh et al., 2012). Para el tercer factor se mantuvo el constructo Distracción (Heydecke et al., 2004; Krägeloh et al., 2012). En este caso, los CPI tomaban tiempo para distraerse; decían que visitar familiares con los cuales podían expresarse emocionalmente sobre su situación como cuidadores les ayudaba.

El caso del cuarto factor, denominado Planificación activa, fue similar a lo reportado anteriormente por Krägeloh et al. (2012) y Pozzi et al. (2015), y se constituyó con ítems de afrontamiento activo denotando comportamientos de planeación y esfuerzo ante las actividades de cuidado. Por su parte, la Reformulación positiva se estructuró en un mismo factor; estos ítems de la subescala no se separaron, como anteriormente lo han reportado Heydecke et al. (2004), Ornelas et al. (2013),
Pozzi et al. (2015) y Radat et al. (2009) en otras poblaciones. Finalmente, el último factor, llamado Negación, se mantiene en diversas validaciones (Heydecke et al., 2004; Ornelas et al., 2013; Pozzi et al., 2015; Radat et al., 2009) y muestra un esquema cognitivo desadaptativo en esta situación.

Muy raramente se ha hecho un AFC del instrumento, lo que puede deberse a su inestabilidad en las distintas validaciones que se reportan (Brasileiro et al., 2016; Castillo, Marván, Galindo y Landa, 2017; Krägeloh et al., 2012; Reich, Costa y Remor, 2016; Snell, Siegert, Hay-Smith y Surgenor, 2011); sin embargo, en CPI de pacientes con cáncer mexicanos muestra una estructura coherente con la versión original. La presente versión del Inventario de Afrontamiento COPE Breve, en comparación con la validación de Baumstarck et al. (2017), resultó tener mejores propiedades psicométricas.

En conclusión, el Inventario de Afrontamiento COPE Breve es un instrumento válido y confiable que evalúa las estrategias de los CPI para afrontar situaciones estresantes; al ser breve y sencillo de aplicar, es factible utilizarlo en escenarios hospitalarios. Estas características representan un ahorro de recursos materiales y temporales en la evaluación de CPI, y brinda además la posibilidad de mejorar la atención integral del paciente oncológico y de su familia, que es uno de los objetivos prioritarios de la psicooncología.

En combinación con otros instrumentos de evaluación psicológica, la aplicación del COPE Breve permitiría análisis más detallados de las respuestas de afrontamiento que los CPI muestran ante la situación que viven, de modo que el diseño de intervenciones psicológicas para esta población promueva estrategias que contribuyan a disminuir el malestar psicológico generado por el estresor, modificando de este modo aquellas que dificulten la adaptación a la situación estresante de cuidar a un paciente con cáncer.

\section{REFERENCIAS}

Adelman, R.D., Tmanova, L.L., Delgado. D., Dion. S. y Lachs, M.S. (2014). Caregiver burden: A clinical review. Journal of the American Medical Association, 311(10), 1052. Doi: 10.1001/jama.2014.304.

Alexander, S.J. (2010). An intense and unforgettable experience: the lived experience o malignant wounds from the perspectives of patients, caregivers and nurses. International Wound Journal, 7(6), 456-465. 
Propiedades psicométricas del Inventario de Afrontamiento COPE Breve en una muestra de cuidadores primarios informales...

Alfaro R.C., O.I., Morales V., T., Vázquez P., F., Sánchez R., S., Ramos R., B. y Guevara L., U. (2008). Burden, anxiety and depression in patient's caregivers with chronic pain and in palliative care. Revista Médica del Instituto Mexicano del Seguro Social, 46(5), 485-494.

Baumstarck, K., Alessandrini, M., Hamidou, Z., Auquier, P., Leroy, T. y Boyer, L. (2017). Assessment of coping: A new French four-factor structure of the brief COPE inventory. Health and Quality of Life Outcomes, 15(8). Doi: 10.1186/s12955-0160581-9.

Ben-Zur, H., Gilbar, O. y Lev, S. (2001). Coping with breast cancer: Patient, spouse, and dyad models. Psychosomatic Medicine, 63(1), 32-39.

Brasileiro, S., Orsini, M.R., Cavalcante, J., Bartholomeu, D., Montiel, J., Costa P., S.S. y Costa, L.R. (2016). Controversies regarding the psychometric properties of the Brief COPE: The case of the Brazilian-Portuguese version "COPE Breve". PloS One, 11(3), 1-13.

Byrne, B. (2010). Structural equation modeling with AMOS: Basic concepts, applications, and programming. New York: Routledge.

Carver, C. (1997). You want to measure coping but your protocol's too long: consider the brief COPE. International Journal of Behavioral Medicine, 4(1), 92-100.

Carver, C. y Scheier, W.F. (1981). Attention and self-regulation: A control theory approach to human behavior. New York: Springer-Verlag.

Carver, C. y Scheier, M. (1990). Principles of self-regulation: Action and emotion. En E. T. Higgins y R. M. Sorrentino (Eds.): Handbook of motivation and cognition: Foundations of social behavior, vol. 2. (pp. 3-52). New York: The Guilford Press.

Carver, C., Scheier, M. y Weintraub, J. (1989). Assessing coping strategies: a theoretically based approach. Journal of Personality and Social Psychology, 56(2), 267-283.

Castillo, R., Marván, M.L., Galindo, O. y Landa, E. (2017). Afrontamiento en pacientes con cáncer de mama: Una revisión narrativa. Revista Latinoamericana de Medicina Conductual, 8(1), 35-42.

Doron, J., Trouillet, R., Gana, K., Boiché, J., Neveu, D. y Ninot, G. (2014). Examination of the hierarchical structure of the brief COPE in a French sample: Empirical and theoretical convergences. Journal of Personality Assessment, 96(5), 567-575.

Espinoza, F., Reyes, I., Velasco, E., Bravo, M. y Ramírez, A. (2017). Escala de estrategias de enfrentamento para cuidadores primarios informales de pacientes con cáncer. Psicooncología, 14(1), 121-136.

Feldberg, C., Tartaglini, M.F., Clemente, M.A., Petracca, G., Cáceres, F. y Stefani, D. (2011). Vulnerabilidad psicosocial del cuidador familiar. Creencias acerca del estado de salud del paciente neurológico y el sentimiento de sobrecarga. Neurología Argentina, 3(1), 11-17.

Fillion, L., Kovacs, A., Gagnon, P. y Endler, N. (2002). Validation of the shortened COPE for use with breast cancer patients undergoing radiation therapy. Current Psychology, 21(1), 17-34.

García C., M., Mateo R., I. y Maroto N., G. (2004). El impacto de cuidar en la salud y la calidad de vida de las mujeres. Gaceta Sanitaria, 18(5), 83-92.

Gardner, R.C. y Escalona, R. (2003). Estadística para psicología: Usando SPSS para Windows. Madrid: Pearson Educación.

George, D. y Mailery, M. (2001). Using SPSS for Windows step by step: a simple guide and reference. New York: Allyn \& Bacon.

Hair, J.F., Anderson, R.E., Tatham, R.L. y Black, W.C. (1999). Análisis multivariante. Madrid: Iberia Prentice-Hall.

Hastings, R.P., Kovshoff, H., Brown, T., Ward, N.J., Espinosa, F.D. y Remington, B. (2005). Coping strategies in mothers and fathers of preschool and school-age children with autism. Autism: The International Journal of Research and Practice, 9(4), 377-391.

Hernández, R., Fernández, C. y Baptista, P. (2014). Metodología de la investigación (6a ed.). México: McGraw-Hill Education.

Heydecke, G., Tedesco, L.A., Kowalski, C. y Inglehart, M. R. (2004). Complete dentures and oral health-related quality of life-Do coping styles matter? Community Dentistry and Oral Epidemiology, 32(4), 297-306.

Hu, L.T. y Bentler, M. (1998). Fit indices in covariance structure modeling: Sensitivity to underparameterized model misspecification. Psychological Methods, 3(4), 424.

Hudson, P., Aranda, S. y Kristjanson, L. (2004). Meeting the supportive needs of family caregivers in palliative care: Challenges for health professionals. Journal of Palliative Medicine, 7(1), 19-26.

Hudson, P. y Payne, S. (2009). Family carers in palliative care: a guide for health and social care professionals. Oxford, UK: Oxford University Press.

Kapsou, M., Panayiotou, G., Kokkinos, C. y Demetriou, A. (2010). Dimensionality of coping: An empirical contribution to the construct validation of the brief-COPE with a Greek-speaking sample. Journal of Health Psychology, 15(2), 215-229.

Kimemia, M., Asner-Self, K.K. y Daire, A.P. (2011). An exploratory factor analysis of the brief cope with a sample of Kenyan caregivers. International Journal for the Advancement of Counselling, 33(3), 149-160.

Kline, R. (2005). Principles and practice of structural equation modeling. New York: The Guilford Press. 
Krägeloh, C.U., Chai, P.P.M., Shepherd, D. y Billington, R. (2012). How religious coping is used relative to other coping strategies depends on the individual's level of religiosity and spirituality. Journal of Religion and Health, 51(4), 1137-1151. Doi: 10.1007/s10943-010-9416-x.

Kristjanson, L.J., Aoun, S. (2004). Palliative care for families: remembering the hidden patients. Canadian Journal of Psychiatry, 49(6), 359-365.

Kvillemo, P. y Bränström, R. (2014). Coping with breast cancer: A meta-analysis. Plos One, 9(11), 1-26.

Lamura, G., Mnich, E., Wojszel, B., Nolan, M., Krevers, B., Mestheneos, L., Döhner, H. y EuroFamCare-Konsortium (2006). The experience of family carers of older people in the use of support services in Europe: Selected findings from the EuroFamCare Project. Zeitschrift Fur Gerontologie Und Geriatrie, 39(6), 429-442. Doi: 10.1007/s00391-006-0416-0.

Larrañaga, I., Martín, U., Bacigalupe, A., Begiristáin, J.M., Valderrama, M. y Arregui, B. (2008). Impacto del cuidado informal en la salud y la calidad de vida de las personas cuidadoras: análisis de las desigualdades de género. Gaceta Sanitaria, 22(5), 443-450. Doi: 10.1157/13126925.

Lazarus, R.S. y Folkman, S. (1984). Stress, appraisal, and coping (11a ed.). New York: Springer.

Lee, R.M. y Liu, H.-T.T. (2001). Coping with intergenerational family conflict: Comparison of Asian American, Hispanic, and European American college students. Journal of Counseling Psychology, 48(4), 410-419. Doi: 10.1037/0022-0167.48.4.410.

Maroco, J., Campos, J.B., Bonafé, F.S., Vinagre, M. y Pais-Ribeiro, J. (2014). Transcultural adaptation Brazil-Portugal of the Brief Cope Scale for college students. Psicologia, Saúde y Doenças, 15(2), 300-313. Doi: 10.15309/14psd150201.

Marsh, H.W., Balla, J.R. y McDonald, R.P. (1988). Goodness-of-fit indexes in confirmatory factor analysis: The effect of sample size. Psychological Bulletin, 103(3), 391-410.

Martínez, E., Díaz, F. y Gómez, E. (2010). Sobrecarga del cuidador primario que asiste a un familiar con cáncer. Altepepaktl, $6(11), 32-41$.

McMillan, S.C., Small, B.J., Weitzner, M., Schonwetter, R., Tittle, M., Moody, L. y Haley, W.E. (2006). Impact of coping skills intervention with family caregivers of hospice patients with cancer: A randomized clinical trial. Cancer, 106(1), 214-222.

Mohanraj, R., Jeyaseelan, V., Kumar, S., Mani, T., Rao, D., Murray, K.R. y Manhart, L.E. (2015). Cultural adaptation of the Brief COPE for persons living with HIV/AIDS in southern India. AIDS and Behavior, 19(2), 341-351.

Morán, C., Landero, R. y González, M. (2010). COPE-28: un análisis psicométrico de la versión en español del Brief COPE. Universitas Psychologica, 9(2), 543-552.

National Alliance for Caregiving (2015). Caregiving in the U.S. 2015. Washington, D.C.: NAC.

Nigenda, G., López O., M., Matarazzo, C. y Juárez R., C. (2007). La atención de los enfermos y discapacitados en el hogar: retos para el sistema de salud mexicano. Salud Pública de México, 49(4), 286-294.

Nijboer, C., Tempelaar, R., Sanderman, R., Triemstra, M., Spruijt, R. y Van den Bos, G. (1998). Cancer and caregiving: the impact on the caregiver's health. Psycho-Oncology, 7(1), 3-13.

Ornelas, R., Tufiño, M., Sierra, A., Guerrero, O., Rosas, A. y Sánchez-Sosa, J.J. (2013). Afrontamiento en pacientes con cáncer de mama en radioterapia: Análisis de la Escala COPE Breve. Psicología y Salud, 23(1), 55-62.

Ortega A., P., Galindo V., O. y Meneses G., A. (2018). Factores ambientales y estrés en salas de espera: comparación en cuidadores primarios informales de pacientes con cáncer. Revista Latinoamericana de Medicina Conductual, 9(1), 41-50.

Osse, B., Vernooij-Dassen, M., Schadé, E. y Grol, R. (2006). Problems experienced by the informal caregivers of cancer patients and their needs for support. Cancer Nursing, 29(5), 378-388.

Papastavrou, E., Charalambous, A. y Tsangari, H. (2009). Exploring the other side of cancer care: the informal caregiver. European Journal of Oncology Nursing, 13(2), 128-136.

Papastavrou, E., Tsangari, H., Karayiannis, G., Papacostas, S., Efstathiou, G. y Sourtzi, P. (2011). Caring and coping: the dementia caregivers. Aging \& Mental Health, 15(6), 702-711.

Pilatti, A., Godoy, J.C. y Brussino S., A. (2012). Adaptación de instrumentos entre culturas: Ejemplos de procedimientos seguidos para medir las expectativas hacia el alcohol en el ámbito argentino. Trastornos Adictivos, 14(2), 58-64.

Pozzi, G., Frustaci, A., Tedeschi, D., Solaroli, S., Grandinetti, P., Di Nicola, M. y Janiri, L. (2015). Coping strategies in a sample of anxiety patients: Factorial analysis and associations with psychopathology. Brain and Behavior, 5(8), 1-10.

Printz, C. (2011). Cancer caregivers still have many unmet needs. Cancer, 117(7), 1331-1333.

Radat, F., Lantéri-Minet, M., Nachit-Ouinekh, F., Massiou, H., Lucas, C., Pradalier, A., Mercier, F. y El Hasnaoui, A. (2009). The GRIM2005 study of migraine consultation in France. III: Psychological features of subjects with migraine. Cephalalgia: An International Journal of Headache, 29(3), 338-350.

Reich, M., Costa Ball, C.D. y Remor, E. (2016). Estudio de las propiedades psicométricas del Brief COPE para una muestra de mujeres uruguayas. Avances en Psicología Latinoamericana, 34(3), 615-636.

Rhee, Y.S., Yun. Y.H., Park, S., Shin, D.O., Lee, K.M., Yoo, H.J., Kim, J.H., Kim, S.O., Lee, R., Lee, Y.O. y Kim, N.S. (2008). Depression in family caregivers of cancer patients: The feeling of burden as a predictor of depression. Journal of Clinical Oncology, 26(36), 5890-5895. 
Propiedades psicométricas del Inventario de Afrontamiento COPE Breve en una muestra de cuidadores primarios informales...

Ruiz, R.J., Gennaro, S., O’Connor, C., Marti, C.N., Lulloff, A., Keshinover, T., Gibeau, A. y Melnyk, B. (2015). Measuring coping in pregnant minority women. Western Journal of Nursing Research, 37(2), 257-275.

Snell, D.L., Siegert, R.J., Hay-Smith, E.J.C. y Surgenor, L.J. (2011). Factor structure of the brief COPE in people with mild traumatic brain injury. The Journal of Head Trauma Rehabilitation, 26(6), 468-477.

Su, X., Lau, J.T., Mak, W.W., Choi, K., Feng, T., Chen, X., Liu, C., Liu, J., Liu, D., Chen, L., Song, J., Zhang, Y., Zhao, G., Zhu, Z. y Cheng, J. (2015). A preliminary validation of the Brief COPE instrument for assessing coping strategies among people living with HIV in China. Infectious Diseases of Poverty, 4(1), 1-10.

Ullgren, H., Tsitsi, T., Papastavrou, E. y Charalambous, A. (2018). How family caregivers of cancer patients manage symptoms at home: A systematic review. International Journal of Nursing Studies, 85, 68-79.

Ullman, J.B (2006). Structural equation modeling: Reviewing the basics and moving forward. Journal of Personality Assessment, $87(1), 35-50$

van Ryn, M., Sanders, S., Kahn, K., van Houtven, C., Griffin, J.M., Martin, M., Atienza, A. A., Phelan, S., Finstad, D. y Rowland, J. (2011). Objective burden, resources, and other stressors among informal cancer caregivers: A hidden quality issue? Psycho-Oncology, 20(1), 44-52.

Vanderwerker, L.C., Laff, R.E., Kadan-Lottick, N.S., McColl, S. y Prigerson, H.G. (2005). Psychiatric disorders and mental health service use among caregivers of advanced cancer patients. Journal of Clinical Oncology, 23(28), 6899-6907.

Vaquiro, S. y Stiepovich, J. (2010). Cuidado informal, un reto asumido por la mujer. Ciencia y Enfermería, 16(2), 9-16.

Vargas, S., Herrera, G.P., Rodríguez, L. y Sepúlveda, G. (2010). Confiabilidad del Brief cope Inventory en versión en español para evaluar estrategias de afrontamiento en pacientes con cáncer de seno. Investigación en Enfermería: Imagen y Desarrollo, 12(1), 1-18.

Vázquez O., G., García A., M., Gómez Á., H., Galeana P., C., Bernal L., S., Rivera F., L. y Ponce J., L.A. (2017). Propiedades psicométricas del cuestionario general del salud de Goldberd-QHQ-28-en cuidadores primarios informales de pacientes con cáncer. Psicooncología, 14(1), 71-84.

Wenk, R. y Monti, C. (2006). Evaluación del sufrimiento de los cuidadores responsables de pacientes con cáncer asistidos en un centro de cuidados paliativos en Argentina. Medicina Paliativa, 13(2), 64-68.

Yusoff, N., Low, W.Y. y Yip, C.H. (2010). Reliability and validity of the Brief COPE Scale (English version) among women with breast cancer undergoing treatment of adjuvant chemotherapy: A Malaysian study. The Medical Journal of Malaysia, $65(1), 41-44$

Zelikovsky, N., Schast, A.P. y Jean-Francois, D. (2007). Parent stress and coping: waiting for a child to receive a kidney transplant. Journal of Clinical Psychology in Medical Settings, 14(4), 320-329. 